\title{
Simultaneous Tune and Coupling Feedback in RHIC, and Possible Implications for LHC Commissioning
}

Peter Cameron, A. DellaPenna, L. Hoff, Y. Luo, A. Marusic, C. Schultheiss, S. Tepikian, BNL Marek Gasior, Rhodri Jones, CERN

Cheng-Yang Tan, FNAL

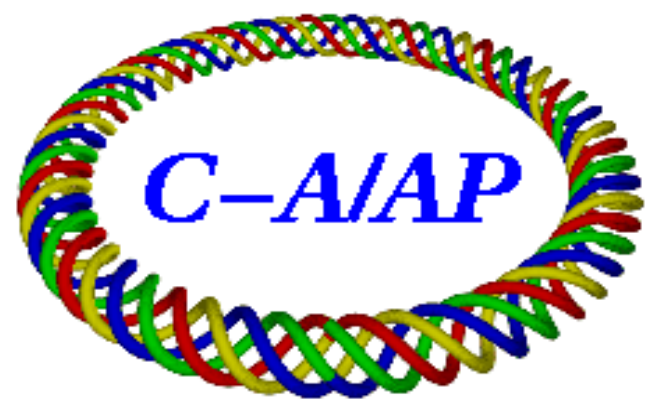

Collider-Accelerator Department Brookhaven National Laboratory Upton, NY 11973 


\title{
Simultaneous Tune and Coupling Feedback in RHIC, and Possible Implications for LHC Commissioning
}

\author{
P. Cameron, A. DellaPenna, L. Hoff, Y. Luo, A. Marusic, C. Schultheiss, and \\ S. Tepikian \\ Brookhaven National Laboratory, Upton, NY, USA 11973 \\ M. Gasior and R. Jones \\ CERN, European Organization for Nuclear Research, CH-1211 Geneva 23, Switzerland \\ C.Y. Tan \\ Tevatron Department, Fermi National Accelerator Laboratory, Illinois, USA
}

(Dated: July 16, 2006)

\begin{abstract}
The Relativistic Heavy Ion Collider (RHIC) is a versatile facility, capable of operating with polarized protons and a variety of ion species over a broad range of energies. Acceleration ramp requirements change frequently to meet the evolving needs of the physics experiments. There has been an ongoing effort at RHIC [1] to implement reliable betatron tune feedback as a tool for ramp development, and possibly to control tune during routine operations. Earlier efforts [2,3] were hampered by large betatron coupling, as well as the requirement for large dynamic range. Both problems have been addressed, the first by continuous measurement of coupling $[4,5]$, and the second by an improved analog front end [6,7]. With these improvements, simultaneous tune and coupling feedback were successfully implemented during RHIC Run 6. In this paper we describe the measurement and control hardware and software used to accomplish this, present some of the results, discuss areas that require further investigation, and finally offer a few comments on possible implications of these results for LHC commissioning.
\end{abstract}

PACS numbers: 2920Dh, 2927Eg, 2927Fh, 4185Qg 


\section{INTRODUCTION}

In hadron accelerators, to minimize emittance growth and the consequent reduction in luminosity available to the physics experiments, the beam excitation needed to reliably operate phase-locked loop (PLL) tune measurement systems must be very small. The power in the resulting betatron signal is typically of the order of femtowatts, while the power delivered to the pickup unrelated to the betatron tune is of the order of watts. In addition, transition crossing in RHIC introduces complications resulting from bunch shortening and fast orbit, tune and chromaticity changes. Much effort was devoted to solving this problem of dynamic range, which was ultimately dealt with by the Direct Diode Detection Analog Front End (3D AFE).

PLL tune tracking and feedback become impossible as coupling becomes large. Coupling rotates the planes of betatron oscillation (the eigenmodes) away from the planes in which the magnet portion of a tune feedback loop applies corrections. When this rotation exceeds 45 degrees the magnet loop applies corrections in the wrong plane and the feedback is driven unstable. To deal with this, additional data acquisition channels were added to the PLL to measure the amplitude and phase of both eigenmode projections in both planes, making possible continuous coupling measurement, and eventually feedback.

These improvements were incorporated into a new VME-based tune and coupling measurement and feedback system that was assembled for RHIC Run 6. The system performed as expected 'out of the box', tracking tunes and measuring coupling within minutes of the first circulating bunched beam.

The two RHIC rings are cooled to helium temperature sequentially, with about one week elapsing between the time when the first 'blue' and second 'yellow' rings are cold. During the set-up period of that one week, preliminary tuning of the PLL system and integration with Controls and the Magnet system was completed. At the end of the week, with both rings cold, ramping commenced. The first ramp was done with feedbacks off, to establish a baseline. For the next ramp feedbacks were turned on, and decoupled beam was delivered to full energy. To the best of our knowledge, this 
successful ramp was the world's first attempt at beam acceleration in a synchrotron with simultaneous tune and coupling feedback.

\section{COUPLING FORMALISM}

A brief description of the coupling formalism [8] is presented here, to assist in understanding material presented later in this paper. Figure 1 shows the eigenmodes and their projections. In the most general case the eigenmodes are ellipses, which are not orthogonal to each other, as shown in the right portion of the figure. The left portion shows the special case when the minor axes of the ellipses vanish. The frequency of mode 1 is denoted by $Q_{1}$, while $A_{1, x}$ and $A_{1, y}$ represent the amplitudes of this mode in the horizontal and vertical plane respectively. Similarly $\phi_{1, \mathrm{x}}$ and $\phi_{1, \mathrm{y}}$ represent the phases of this mode in the horizontal and vertical plane respectively. The same notation applies for mode 2 .
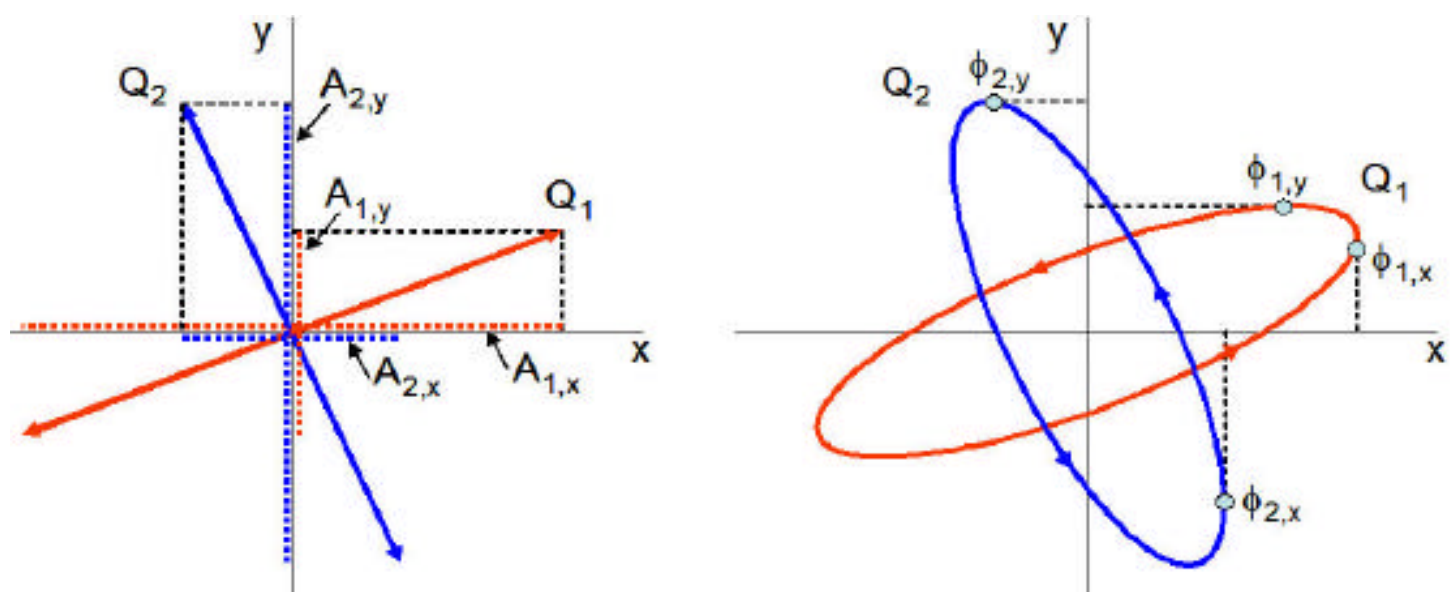

FIGURE 1. (color) Schematics showing the two eigenmodes rotated with respect to the horizontal and vertical planes due to coupling

All of the quantities shown in the figure are measured by the PLL. From this data the following parameters can be calculated:

$$
\begin{gathered}
r_{1}=\frac{A_{1, y}}{A_{1, x}}=\sqrt{\frac{\beta_{y}}{\beta_{x}}} \cdot \frac{\left|C^{-}\right|}{2 v+\Delta}, r_{2}=\frac{A_{2, x}}{A_{2, y}}=\sqrt{\frac{\beta_{x}}{\beta_{y}}} \cdot \frac{\left|C^{-}\right|}{2 v+\Delta}, \\
d \phi_{1}=\phi_{1, y}-\phi_{1, x}, d \phi_{2}=\phi_{2, x}-\phi_{2, y}
\end{gathered}
$$

where $\beta_{\mathrm{x}}$ and $\beta_{\mathrm{y}}$ are the horizontal and vertical beta functions at the pickup, and 


$$
v=\frac{1}{2} \sqrt{\Delta^{2}+\left|C^{-}\right|^{2}},\left|C^{-}\right|=\frac{2 \sqrt{r_{1} r_{2}}\left|Q_{1}-Q_{2}\right|}{\left(1+r_{1} r_{2}\right)}, \Delta=\frac{\left|Q_{1}-Q_{2}\right|\left(1-r_{1} r_{2}\right)}{\left(1+r_{1} r_{2}\right)}
$$

When the eigenmodes are decoupled they are in the horizontal and vertical planes, the ratios $r_{1}$ and $r_{2}$ are equal to zero, the ellipses collapse to straight lines, and the coupling phases $d \phi_{1}$ and $d \phi_{2}$ are equal to zero. The coupling amplitude $\mathrm{C}^{-}$is often referred to as $\mathrm{dQ}_{\min }$, or the minimum tune split. The parameter $\Delta$ is the difference between the fractional parts of the unperturbed, or 'set' tunes. When the eigenmodes are decoupled the eigentunes (measured) and the unperturbed (set) tunes are identical. For the purpose of presenting the data in a form that is easily understood, the unperturbed tunes can be calculated by:

$$
Q_{x, 0}=Q_{1}+\frac{1}{2} \Delta-\frac{1}{2} \sqrt{\Delta^{2}+\left|C^{-}\right|^{2}}, Q_{y, 0}=Q_{2}-\frac{1}{2} \Delta+\frac{1}{2} \sqrt{\Delta^{2}+\left|C^{-}\right|^{2}}
$$

The utility of having available both set and measured tunes will become clear later in this paper.

\section{SYSTEM ARCHITECTURE AND SIGNAL PROCESSING}

Figure 2 shows a block diagram of the tune and coupling measurement and feedback system. Analog processing of signals from the pickup is accomplished by the 3D AFE. After detection, filtering, and amplification the tune signals from the two pickup planes are split and digitized. Digital processing is accomplished in two signal paths that, with the exception of coupling correction, are independent. The two signal paths provide the data needed needed to maintain phase lock to the two eigenmodes, and to correct coupling and tune, and eventually chromaticity.

When the eigenmode PLLs are locked, the digitizer clocks are phase-synchronous to the eigenmodes. As shown in the figure, the digitizer clocks are generated by numerically-controlled oscillators (NCOs), one for each eigenmode. The NCOs are beam-synchronously clocked from the $28 \mathrm{MHz}$ RF. Their output is at four times the betatron frequency, permitting simple I/Q demodulation. 


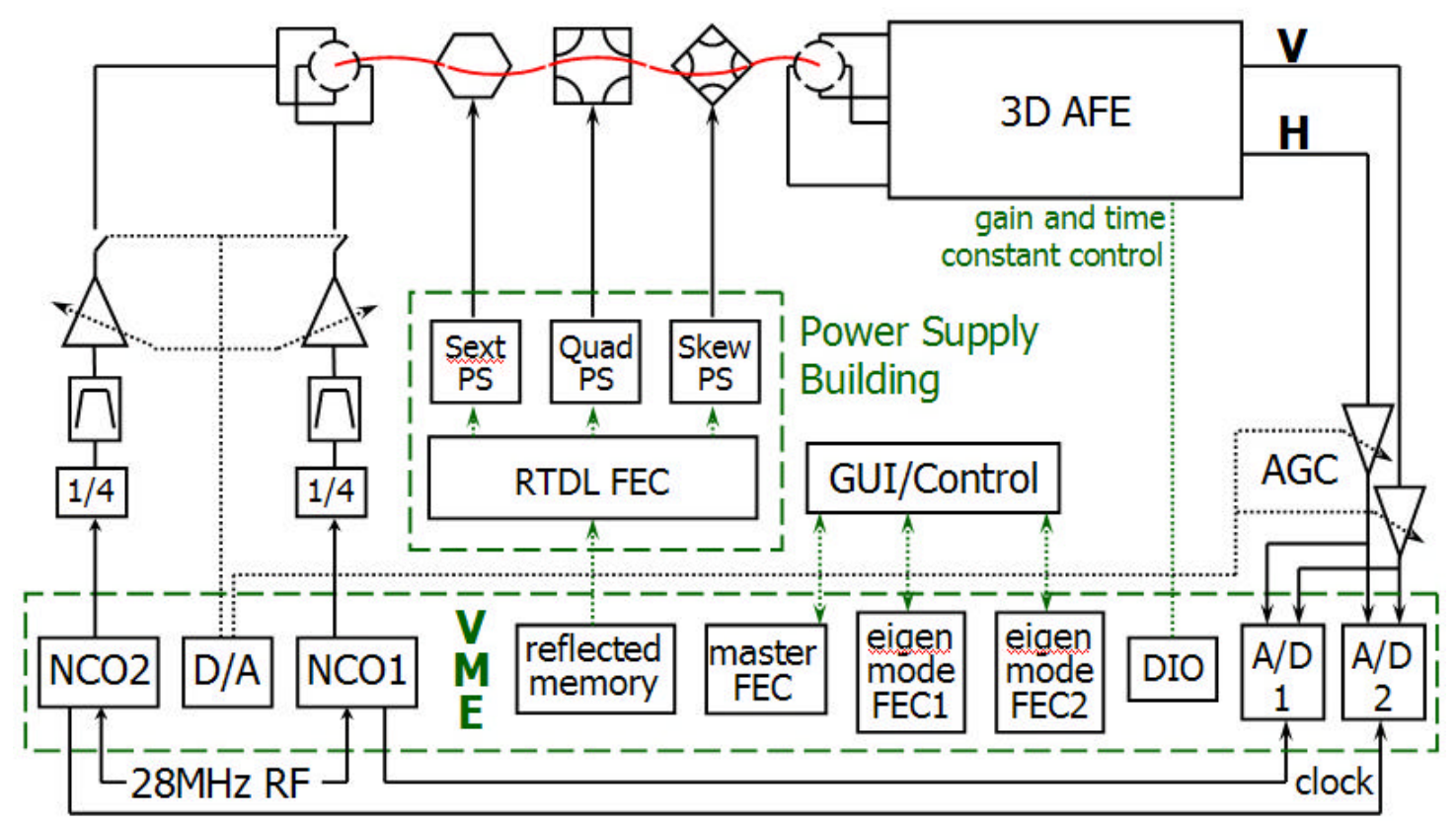

FIGURE 2. (color) System Block Diagram

Data for each eigenmode is processed by a Front End Computer (FEC) dedicated to that eigenmode. Processed data is passed to a Master FEC that calculates magnet correction strengths. Corrections are written to a reflected memory module that is mirrored via dedicated fiber to a similar module in the power supply building, where a FEC applies the corrections to the power supplies. Kicker and signal path gains, as well as the 3D AFE gains and time constants, are controlled by additional VME modules. The RHIC implementation accomplishes measurement and control for both the 'blue' and 'yellow' rings in a single VME crate, and the number of VME modules in that crate is approximately twice that shown in the diagram.

\section{A. Kicker and Pickup}

The kicker design originated at CERN, and was driven by two considerations. The first was the need to excite the the rigid $7 \mathrm{TeV}$ LHC beam without excessive kicker power, and second was to couple the kicker effectively to the beam without presenting excessive impedance to the beam when viewed in its reciprocal role as a pickup.

Figure 3 shows the relative contributions of the electric and magnetic fields to the total field in the pickup as a function of the kicker impedance, at constant power. It is clearly advantageous to favor the magnetic field, and to operate at the lowest practical 
impedance. The PLL operates at baseband, where transmission line effects can be ignored, and the minimum practical impedance is then defined by the resistance of the cables, connectors, feedthrus, and striplines, as well as by the current carrying capability of the feedthrus. This approach eases the task of exciting the beam without excessive kicker power.

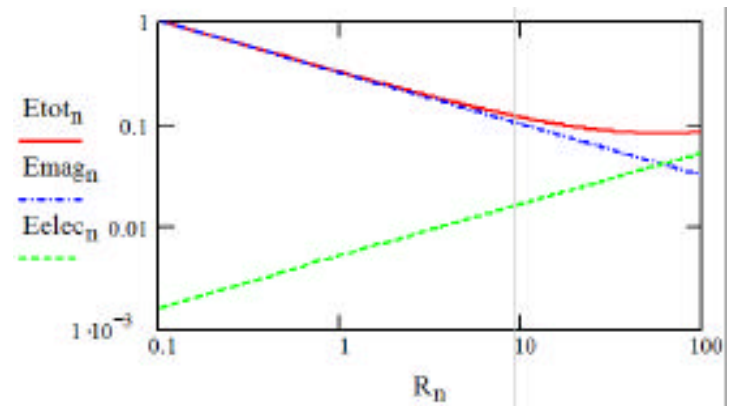

FIGURE 3. Kick as a function of impedance

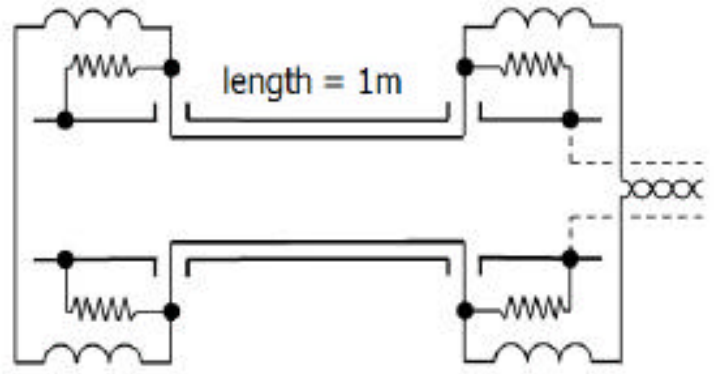

FIGURE 4. Kicker block diagram

Figure 4 shows the kicker layout adopted for RHIC. The kicker is a $1 \mathrm{~m}$ long dual plane device. The $50 \Omega$ striplines subtend 70 degrees, and their inside diameter is $11 \mathrm{~cm}$. The design exploits the fact that kickers and pickups are not truly reciprocal devices[9,10]. A kicker has response at DC, but a pickup does not. The kicker is driven in series thru the four $1 \mu \mathrm{H}$ inductors shown in the figure. Their impedance is $\sim 0.1 \Omega$ at the $\sim 20 \mathrm{KHz}$ betatron frequency of RHIC, but more like $400 \Omega$ at the $75 \mathrm{MHz}$ response peak of the $1 \mathrm{~m}$ stripline when viewed as a pickup. The inductors permit low impedance driving of the device as a kicker, and effectively block its response as a pickup, protecting the driver amplifier. The $50 \Omega$ resistors shown in the figure provide a matched termination for the pickup response of the striplines.

The kicker is driven differential mode through shielded twisted pairs. The system has been operated both with the 3D AFE in the tunnel, and with beam signals cabled out on heliax. There are no problems with bleedthru across the $\sim 160 \mathrm{~dB}$ difference between the kicker path and the signal path, as long as single point grounding is maintained with the beampipe serving as ground.

The pickup is identical in construction to the kicker. Signal is taken from the upstream ports, and the downstream ports are terminated in $50 \mathrm{ohms}$. Beta functions at the pickup are $\beta_{\mathrm{h}} \sim 25 \mathrm{~m}$ and $\beta_{\mathrm{y}} \sim 130 \mathrm{~m}$. Motion control is available for centering the 
pickup on the beam if so desired. Our experience so far has been that beam centering in the pickup is not critical, and motion feedback has not been implemented.

\section{B. Direct Diode Detection Analog Front End}

The crucial part of the 3D AFE is a peak detector. Two such detectors connected to opposing electrodes of the pickup, as shown in Figure 5, yield the amplitude modulation envelope of the beam signals. Such signals, depicted in Figure 6, are superimposed on a DC voltage related to the bunch amplitude. The signal difference, shown in Figure 7 for a single bunch in the machine, contains almost the whole bunch modulation amplitude, with a DC value related to the beam offset from the center of the pick-up. Since the DC content can be suppressed using a series capacitor, the corresponding revolution frequency $\left(f_{r}\right)$ background can already easily be removed by some $50 \mathrm{~dB}$ before the first amplifying stage.
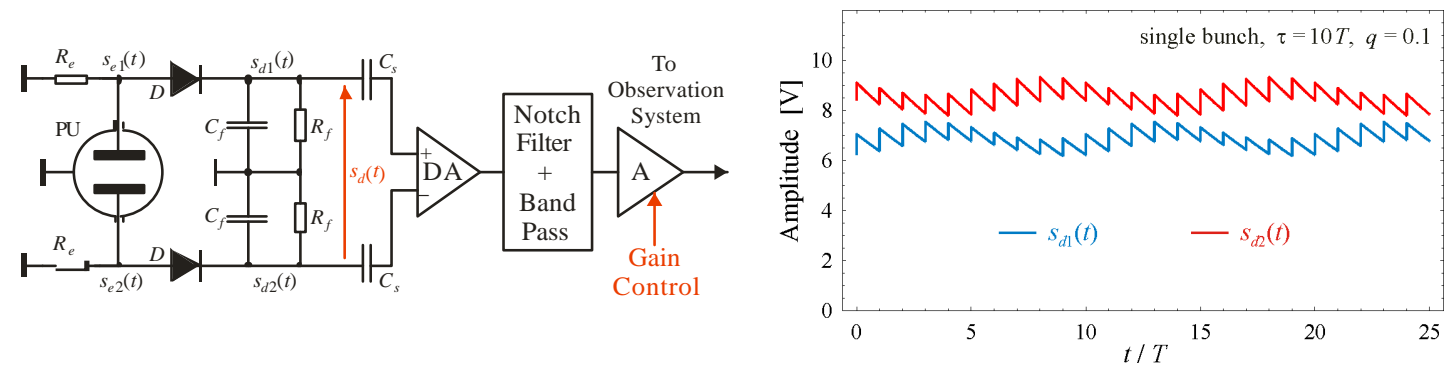

FIGURE 5. Block diagram of a 3D AFE

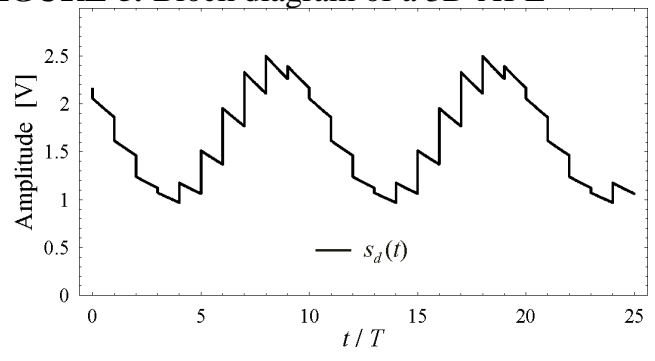

FIGURE 6. Example of peak detector voltages

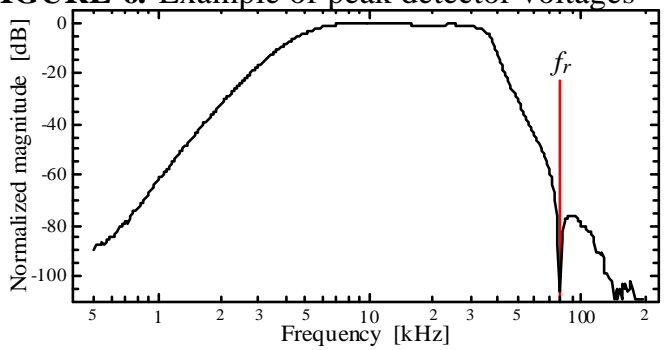

FIGURE 7. Difference of the signals in figure 6

FIGURE 8. RHIC AFE frequency characteristic

The 3D circuitry in Figure 5 can be thought of as two sample-and-hold blocks, sampling bunch signals close to their maxima at the bunch repetition rate and downmixing the wideband bunch spectrum into the baseband. Note that a $3 \mathrm{D}$ AFE is "low frequency" only after the detectors, due to the "time stretching" of the short beam pulses. Before the detectors, the processed bandwidth can extend into the $\mathrm{GHz}$ range, covering the full coherent spectrum in hadron machines. In the detection 
process the spectral content from this wide bandwidth is converted to the baseband, resulting in a system sensitivity in the nm range for high intensity beams.

As sketched in Figure 5, the peak detector voltages with the DC content removed by the series capacitors are subtracted by the differential amplifier (DA). This further increases the suppression of $f_{r}$ and improves the immunity of the system to interference. The filters further attenuate $f_{r}$ by some $100 \mathrm{~dB}$, as seen on the AFE characteristic in Figure 8. The overall $f_{r}$ suppression from all system stages can be conservatively estimated to be some $160 \mathrm{~dB}$. Another advantage of the $3 \mathrm{D}$ approach is that the output signal, which is in the audio range for large machines, can be efficiently digitized with high resolution audio ADCs.

Gain in the 3D AFE is adjustable over a range of $65 \mathrm{~dB}$ in $5 \mathrm{~dB}$ steps. In addition, it is useful to have continuous gain control in both the kicker and signal paths to stabilize the overall system loop gain under varying beam conditions. Provision has been made to accomplish this by monitoring signal strength and the signalto-noise ratio. A feedback loop can be closed in the signal path to maintain constant signal strength, and a similar loop in the kicker path to maintain constant signalto-noise.

\section{PLL Signal Processing}

Commercial-Off-The-Shelf (COTS) components were widely used in assembling the data acquisition and processing systems. Two exceptions are the standard RHIC V108 'utility' module, and the Numerically Controlled Oscillators (NCOs). The V108 module supports a variety of utility functions, such as crate temperature monitoring and decoding RHIC timing and global data signals. Its chief role in the present system is to associate RHIC-specific timestamps to data. The NCO is a standard RHIC module typically used in RF applications. While there may be COTS equivalents, this module has a long and successful track ecord at RHIC in similar applications, and was readily available. The important characteristics for an NCO in this application include appropriate frequency range, support for external clocking to automatically track RF frequency changes during beam energy ramps, and smooth transitions during frequency adjustments to maintain phase continuity. 
The software executes in the standard RHIC software environment. This includes the VxWorks operating system, device drivers (typically written in the " $\mathrm{C}$ " language), and a RHIC-specific abstraction layer called the Accelerator Device Object (ADO) layer, developed using a C++-like language and stored in files called 'rad files'.

\begin{tabular}{|l|l|l|l|}
\hline Function & Model & Notes & qty \\
\hline ADC & VMIVME 3123 & $\begin{array}{l}\text { 16 channel, 16 bits, external clock, ping- } \\
\text { pong buffers }\end{array}$ & 4 \\
\hline DAC & $\begin{array}{l}\text { VMIVME 4140, } \\
4122\end{array}$ & 8 channel, 16 bits & 3 \\
\hline Digital Output & VMIVME 2170 & 32 channel & 2 \\
\hline CPU & MVME2100 & 200MHz PowerPC 603 & 5 \\
\hline NCO & RHIC RF & External clock & 4 \\
\hline Utility & V108 & Provides timestamps & 1 \\
\hline $\begin{array}{l}\text { Shared } \\
\text { memory }\end{array}$ & MM-6702 & Non-volatile SRAM w/battery backup & 1 \\
\hline $\begin{array}{l}\text { Reflective } \\
\text { memory }\end{array}$ & VMIVME 5576 & Broadcasts calculated magnet corrections & 1 \\
\hline
\end{tabular}

TABLE 1. VME modules used in the tuned feedback system

Hardware modules used in the system are shown in Table 1. Device drivers were already available for all hardware modules, and ADOs were available for most of the needed functions, including basic analog and digital control, utility crate monitoring, timestamp decoding, and even tune feedback (from a previous generation RHIC tune feedback system). Two new ADOs were developed for data acquisition and processing. One of these ADOs supports closed-loop feedback on kicker strength and/or pickup signal gain. The other ADO supports the data acquisition and processing associated with tune and coupling measurement and will be described in detail in the following paragraphs. Four of the $5 \mathrm{CPU}$ modules in the system are assigned to the four eigenmodes of the 2 RHIC rings. Each of these CPUs runs a single instance of the data acquisition ADO and two instances of the feedback ADO, one for kicker strength and one for signal gain. The fifth CPU collects tune and coupling data from the other CPUs, computes tune and coupling correction, and broadcasts those values to magnet power supply controllers. The basic scheme for tune feedback had been demonstrated in a previous generation RHIC tune feedback system. 
The ADC module is configured to continuously acquire data into one of two 'pingpong' buffers. When one buffer is full, the ADC switches to the other buffer and interrupts the CPU, so that data from the first buffer can be processed while the second buffer is being filled. The ADC sampling clock is provided by the NCO, and is adjusted to be four times the betatron frequency, so that the ADC samples contain alternately in-phase and quadrature measurements of beam motion. The number of samples in the ADC buffer is configured to be a multiple of 4 to preserve the relative phase of samples within each of the two buffers.

The ADC interrupt initiates one pass of data processing. During this pass, the ADC data is separated into an average of the in-phase and an average of the quadrature values. Each of these values is inserted into separate data streams. Each of these data streams is then passed through one iteration of a low-pass (FIR) filter. The resulting low-passed quadrature value is then used as the error signal to a PID loop which adjusts the NCO frequency, realizing a PLL locked onto the betatron frequency.

To aid in characterizing the performance of the PLL, certain parameters, including the quadrature (error) signal and the NCO frequency are output as voltages via a third, diagnostic DAC module. The DAC outputs are available for analysis, for instance using a spectrum analyzer.

To measure coupling, the signal from the orthogonal plane is acquired via a second ADC channel, using the same sampling clock. The resulting signal is demodulated using the same technique as the primary channel.

Finally, after decimation several parameters, including the filtered I/Q data from both ADC channels, are published via shared memory so that the fifth (master) CPU can compute the necessary magnet correction strengths to maintain programmed tune and minimize coupling.

\section{Coupling Correction and Magnet Control}

For tune and coupling feedback in both of the RHIC rings, a total of 20 data streams (the 8 eigenmode projections for each of I and Q, plus the 4 tunes) are passed from the 4 eigenmode FECs to the Master FEC, where amplitudes and phases are calculated from the I and Q data. For each projection, the projection amplitude is 
given by $\operatorname{sqrt}\left(\mathrm{I}^{2}+\mathrm{Q}^{2}\right)$. The projection phase is given by $\operatorname{atan} 2(\mathrm{Q}, \mathrm{I})$. If the eigentunes are well tracked, the measure phases $\phi_{1, \mathrm{x}}$ and $\phi_{2, \mathrm{y}}$ are driven to zero by the functioning of the PLL. The phase of the coupling coefficient is simply given by $\phi_{1, y}$. With the eigentune split and the amplitude ratios $\mathrm{rl}$ and $\mathrm{r} 2$, from Eq. (2) the amplitude of coupling coefficient is determined. According to Eq.(3), the uncoupled set tunes are also obtained.

For coupling correction, RHIC has 3 skew quadrupole families, F1, F2, and F3. The coupling contributions from these 3 familes are about 120 degrees apart when they are powered with the same strength and proper polarities. For coupling correction we combine F1 and F3 with the same strength to produce a family F13 whose contribution to the total coupling coefficient is approximately orthogonal to that only from F2 family. Knowing the coupling coefficient at the location of the PLL pickup, based on the accelerator optics model the global decoupling strengths for the two orthogonal families F2 and F13 can be calculated. In reality, the coupling angles of F2 and F13 are slightly modified with the measured phases of the eigenmode projections.

The feedback loop for tune control[11] has the measured tunes as inputs. The differences between the measured and desired tunes are the error signals, from which are calculated the focusing and defocusing quadrupole strength outputs. Implicit in the operation of the tune feedback loop is the assumption that the tunes are decoupled, so that the measured eigentunes correspond to the correction planes of the feedback loop. The two planes typically have different freque ncy responses. The error signals from each plane are filtered separately, permitting the overall loop responses of the two planes to be equalized. A PID filter is utilized as a compensation filter. The magnet feedback loop for coupling control is similar to the tune feedback loop.

\section{RESULTS}

Measurements of the coupling and tune parameters presented in equations (1) through (3) are shown in Figure 9. This data was taken during a tune scan made for working point optimization during RHIC Run 5. Vertical tune was held constant while horizontal tune was scanned. The color scheme used in this figure for set and measured tunes will be followed throughout this paper. In this figure the set and 
measured tunes differ as a result of the minimum tune split due to coupling, and are easily distinguished. In several figures later in this paper the tunes are well decoupled, and the measured tune data is partially or totally obscured by the set tune data.

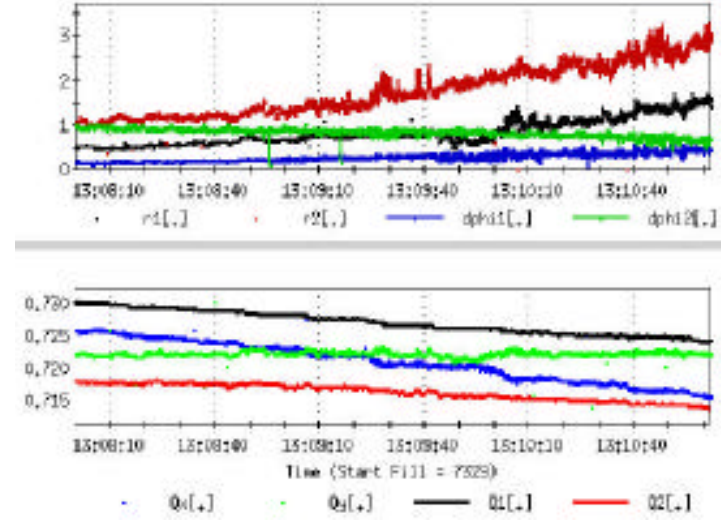

FIGURE 9. (color) Parameters during a tune scan

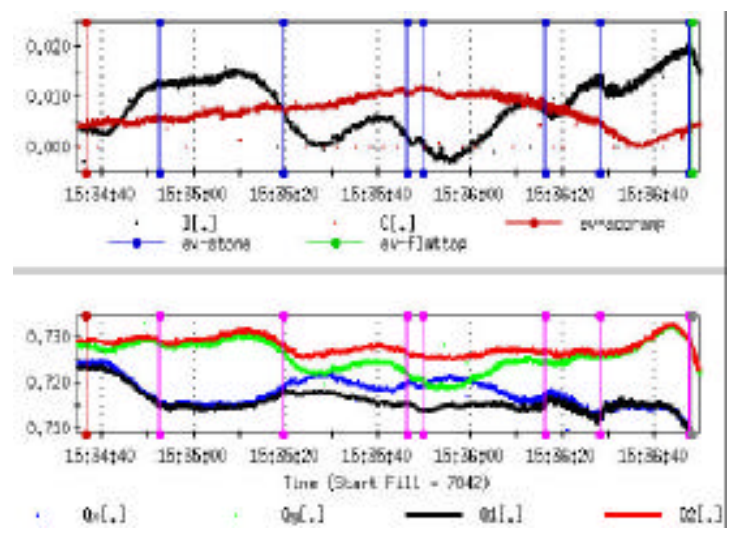

FIGURE 10. (color) Parameters during a ramp

The coupling parameters $\mathrm{C}^{-}$and $\Delta$ are shown in the upper pane of Figure 10, taken from an acceleration ramp during RHIC Run 5. The lower pane shows measured and set tunes, and clearly shows crossing of the set tunes during the ramp. One's understanding of the quality of tune control in both Figure 9 and Figure 10 would be very different without the availability of the coupling data. If tune feedback had been on in these figures, the feedback would have been driven unstable by the crossing of the set tunes.

Success of the tune feedback effort is predicated on decoupling. Therefore, the first objectives when beam became available in Run 6 were to validate the coupling measurement, then close the loop on coupling feedback. Figure 11 shows data taken early in Run 6, during the week of machine setup in preparation for ramp development. The upper pane shows calculated correction strengths for the families F2 and F13, derived from the measured coupling. The lower pane shows set and measured tunes during decoupling. It can be seen that as the requested skew correction strengths are adjusted towards zero, the set tunes converge on the measured tunes.

The commonly accepted fiducial measure of coupling is accomplished by moving the tunes as close as possible to determine the minimum tune split. This method is not often used because it perturbs machine operation, but is useful for comparing results with other measurement methods. From the eigenmode-based coupling measurement, skew strengths can be adjusted to zero, and it can be observed that set and measured 
tunes agree in this case. But the real proof that the tunes are decoupled is to measure the closest tune approach. Figure 12 shows data from a tune scan after the tunes were decoupled via the method shown in Figure 11. It can be seen both that the set and measured tunes were in agreement, and that the closest tune approach was .001 or less.

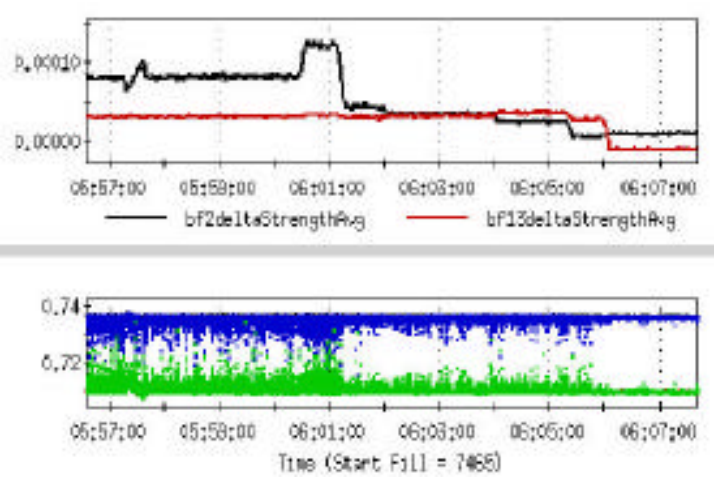

FIGURE 11. (color) Manual de-coupling

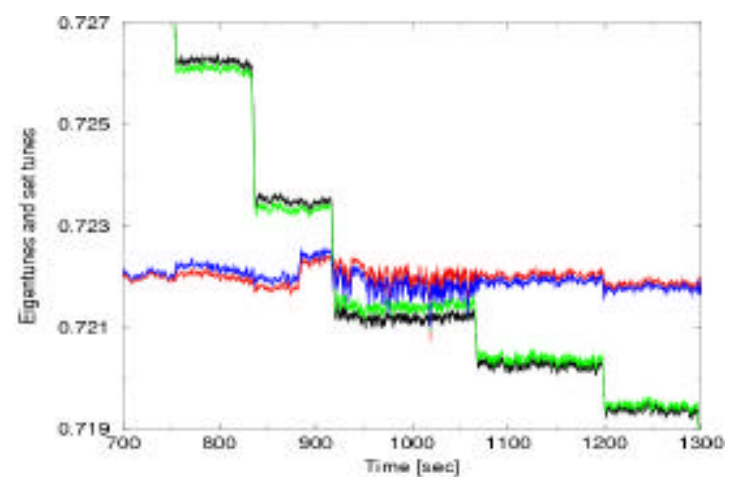

FIGURE 12. (color) Tune scan

Figure 13 shows set and measured tunes as the coupling feedback loop is turned on, then off, and then back on again. With the loop off the measured tunes are driven apart by the coupling. With the loop on the set and measured tunes are essentially the same.

Figure 14 is a bit of a curiousity. During initial setup of the PLL, the the impulse excitation of single kicks from the conventional tune meter kicker were used to tune PID loop parameters. The resulting 'coupling echoes' shown in the figure have two features, the response at twice the synchrotron frequency and the slower response at $\sim 2 \mathrm{~Hz}$. The beam physics responsible for these features is not yet clear.

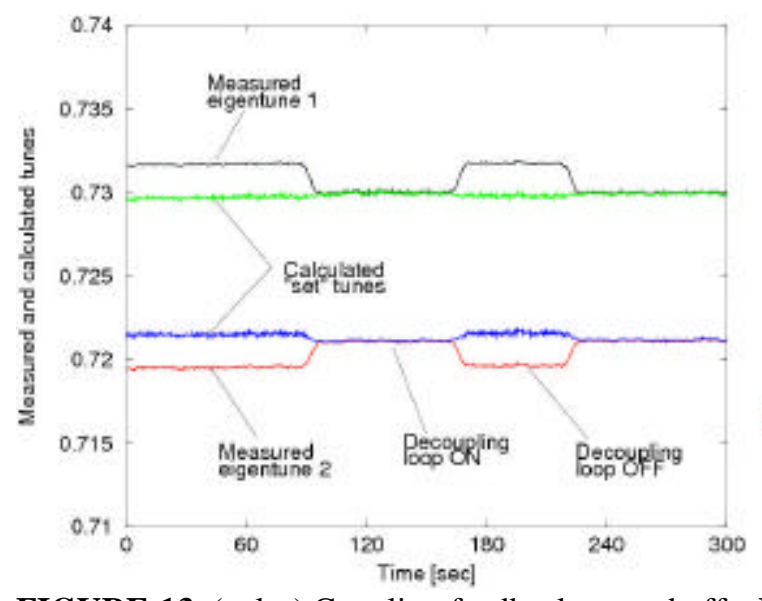

FIGURE 13. (color) Coupling feedback on and off

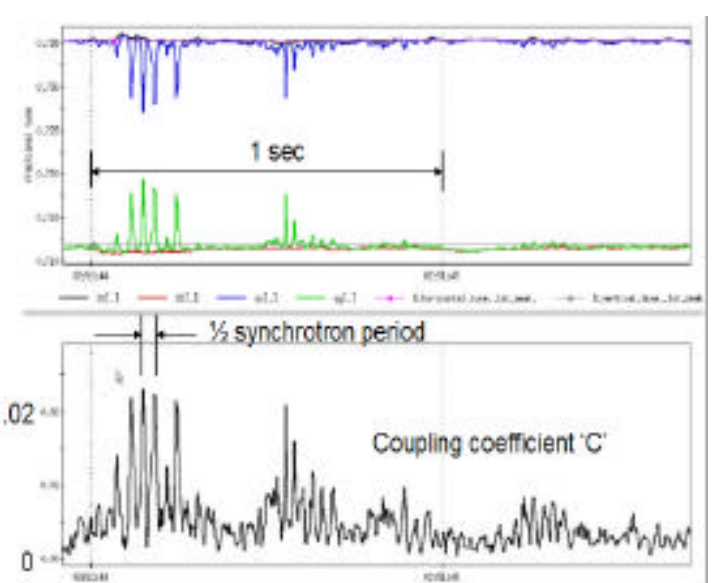

FIGURE 14. (color) Coupling echoes

Figure 15 shows data taken from 3 ramps during $100 \mathrm{GeV}$ ramp development. It should be noted that PLL loop gains must be adjusted manually during ramps with 
feedbacks on, to compensate the effect of chromaticity variations. This will be discussed in more detail in the following section. The uppermost pane in the figure shows blue ring set and measured tunes, as well as beam currents in the blue and yellow rings, during the first acceleration ramp with feedbacks enabled (in the blue ring only). For the most part the correction from the coupling feedback was so good that the measured tune data is obscured by the set tune data. Loop gains in both the PLL tune tracker loop and the overall tune and coupling feedback loops were set somewhat high to insure tracking of fast changes. Additionally, PLL gains were manually adjusted through the ramp to accommodate changing beam conditions.
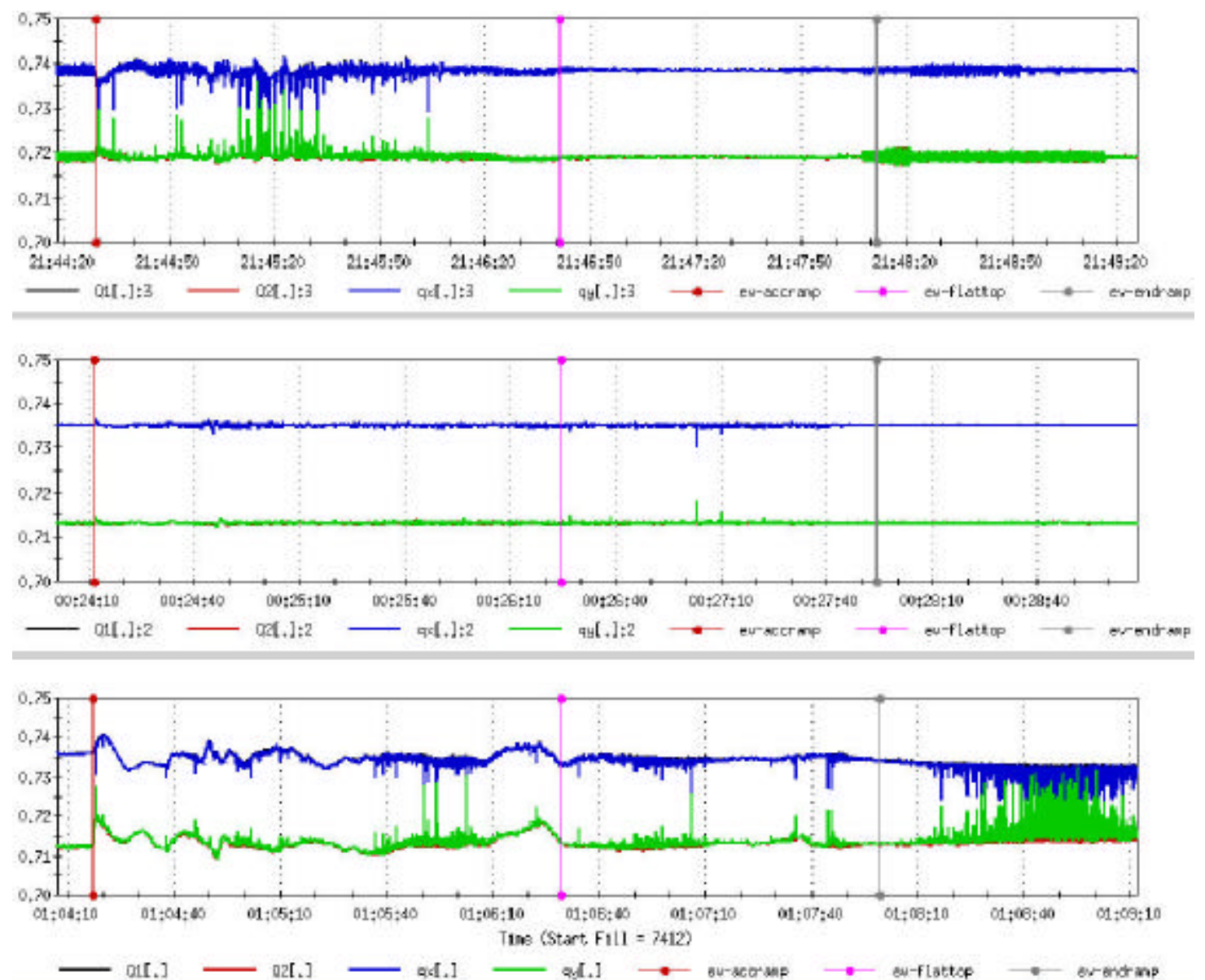

FIGURE 15. (color) Data from $100 \mathrm{GeV}$ development ramps in RHIC Run 6

The middle pane shows the eleventh and last of the $100 \mathrm{GeV}$ feedback ramps in blue. Magnet correction strengths were fed forward from ramp to ramp, so that by the end of this phase of ramp development the required corrections were small, and loop gains could be lowered. The improved quality of the data is clearly evident. 
The lower pane shows data from the next ramp, with feedbacks off and corrections from the previous ramp fed forward. This gives some idea of the ramp-to-ramp variations in tune and coupling. There were no deliberate orbit changes between the two ramps, and hence coupling was not changed significantly.

\section{AREAS FOR FURTHER IN VESTIGATION}

During the course of this work two additional obstacles to making the system fully operational became clear. The first is the presence of large amplitude direct excitation of the betatron resonances by high harmonics ( $h \sim 350$ ) of the $60 \mathrm{~Hz}$ power frequency, and the second is the need for precise chromaticity control (and possibly feedback) for proper operation of the tune tracker.

\section{A. Mains Harmonics}

The 3D AFE delivers significant improvement in position measurement sensitivity, with estimated 10nm resolution at RHIC. This AFE has been installed at the CERN PS and SPS, at the FNAL Tevatron, and at RHIC. Mains harmonics have been observed in the betatron spectrum at every installation [12]. Extensive studies have conclusively deomonstrated that these harmonics result from direct excitation of the beam at the betatron frequency by high harmonics of the power line frequency. At RHIC the resulting spectral lines are as much as $40 \mathrm{~dB}$ above the 3D AFE noise floor at injection and store, and as much as $80 \mathrm{~dB}$ above the noise floor during ramping, when additional supplies are switched in to provide the field time derivative needed for ramping. The source of these harmonics is being actively investigated, and their possible origin is discussed in detail elsewhere [12,13].

\section{B. Chromaticity}

For reliable tune tracking the overall loop gain of the PLL must be relatively constant. The beam-transfer-function portion of the loop gain is dependent on chromaticity. With large chromaticity both the amplitude and the phase slope of the betatron resonance response become small, and loop gain can be diminished to the 
extent that the PLL cannot track fast tune changes. Conversely, with small chromaticity the loop gain can become so large that the loop becomes unstable.

The pickup for previous generations of the RHIC tune feedback system utilized a stripline BPM resonated at $245 \mathrm{MHz}$, above the coherent spectrum. At that frequency the linewidth is dominated by broadening due to the high harmonic number, and studies showed that PLL performance was only weakly affected by chromaticity over the range from approximately 1-20 units. What effect was observed could be sufficiently compensated by feeding back on the kicker excitation to keep the amplitude response constant.

By virtue of its operating principle, the $3 \mathrm{D}$ AFE functions within the coherent spectrum, where the linewidth is dominated by chromaticity. In RHIC the $3 \mathrm{~dB}$ point of the coherent spectrum is at about $60 \mathrm{MHz}$ when beam is bunched in the $28 \mathrm{MHz}$ acceleration cavities. Operational experience suggests that the tolerace of the $3 \mathrm{D}$ AFE PLL to chromaticity variations is less than that of the accelerator itself, and that chromaticity must be well controlled (in the range of 1 to 5 units) for reliable PLL operation. In addition to the expected effects on overall loop gain, the situation is aggravated by the fact the the $3 \mathrm{D}$ AFE functions as a peak detector, and is sensitive to the 'micro-instabilities' that precede the full blown head-tail instability caused by too small chromaticity. As chromaticity becomes small the PLL senses not the kicker excitation, but rather the self-excitations of beam, and loses lock. As previously mentioned, this sensitivity to the effect of chromaticity on overall loop gain required that loop gains be manually adjusted during Run 6 feedback ramps.

Past experience [14] with continuous chromaticity measurement using the PLL has been good. Data taken under automated sequencer control from three successive ramps during Run 5 is shown in Figure 16. This data was taken with the $245 \mathrm{MHz}$ resonant pickup, and the quality of the data near transition suffers due to excessive dynamic range as the bunch length shortens and extends up to the pickup frequency. The 3D AFE functions in the coherent spectrum, and does not have a problem with dynamic range around transition. The measurements were made with radial modulation of the beam with an amplitude of $200 \mu$ (this corresponds to a momentum deviation of $\sim 2 \times 10^{-4}$ ) at a rate of $1 \mathrm{~Hz}$. Excepting the time around transition, the 
measurement appears to be of adequate quality to permit feedback. It should also be mentioned that the algorithm for calculating chromaticity suffers from a sign ambiguity as chromaticity becomes small. This can be seen in the oscillations of the measured horizontal chromaticity around zero near the end of all 3 ramps. The source of this problem remains under investigation. Our expectation is that the quality of chromaticity measurement from the 3D AFE PLL will be similar to or better than that shown in the figure.
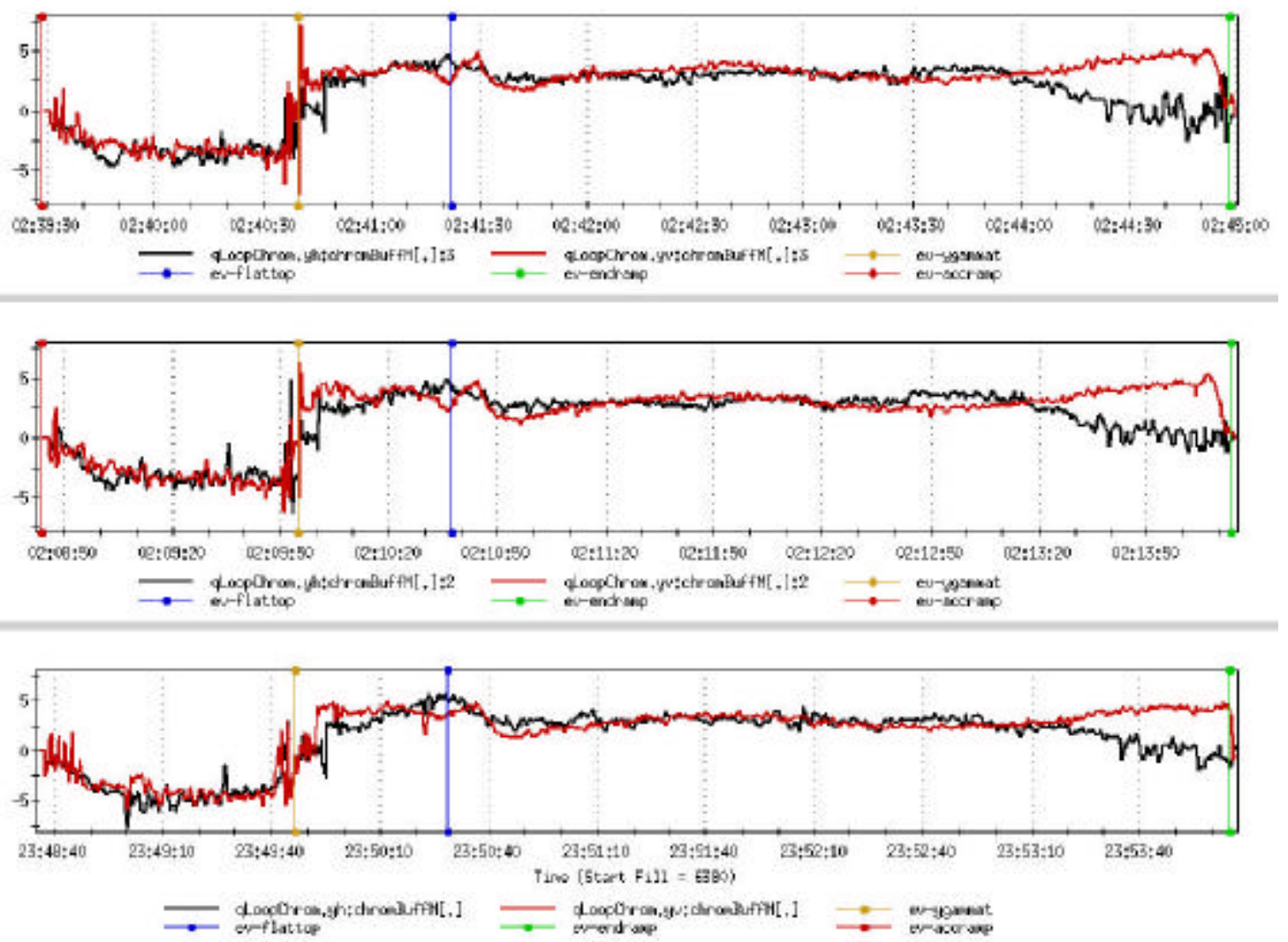

FIGURE 16: (color) Automated chromaticity measurement during three successive ramps

As a result of the observed sensitivity of the PLL to chromaticity, our plan for RHIC Run 7 is to close the last loop, namely chromaticity, in this multi-loop feedback system. We intend to implement simultanous tune, coupling, and chromaticity feedback as early in the run as is feasible. 


\section{POSSIBLE IMPLICATIONS FOR LHC COMMISSIONING}

The 'out of the box' success of simultaneous tune and coupling feedback in RHIC greatly improves the possibility that robust feedbacks can be implemented early in LHC commissioning. From the LHC perspective, the most urgent motivation for feedbacks is management of the chromatic effect of snapback at the beginning of the acceleration ramp [15]. The present understanding is that compensation of this effect will require realtime feedback control of chromaticity. This feedback is moving into the foreground of our efforts, motivated both by LHC needs for snapback control and the need to stabilize PLL loop gain in RHIC.

We emphasize that mains harmonics in RHIC are anomalously large, and their origin is not understood. This has many implications for the LHC. They impede needed system refinement, and obstruct development work on chromaticity measurement and

feedback. Their presence in the beam spectrum introduces spurious phase any time the tune is such that they are within the bandwidth of the PLL, an inevitable circumstance during the radial modulation chromaticity measurement with feedbacks off. They require careful selection of tunes when feedbacks are on. During normal operations with feedbacks off the effects of their spurious excitation diminish the confidence of non-specialist users of the system, impeding its transition to full operational status. And finally, until their origin is understood the possibility cannot be fully eliminated that a similar difficulty might arise in the LHC. Understanding and resolving this problem remains a high priority.

\section{ACKNOWLEDGEMENTS}

The results presented here would not exist without the early foresight and guidance of Tom Shea, and the excellent foundation he provided to support these efforts. We gratefully acknowledge his many contributions.

Hermann Schmickler initiated the collaborative effort between CERN and BNL. He, Mike Harrison and Steve Peggs brought this collaboration into the US LHC Accelerator Research Program (LARP), where it was expanded to include FNAL and has prospered under the oversight of Alex Ratti. The support of the LARP structure 
has been essential from a financial and organizational perspective, and additionally has enhanced the scientific and technical quality of the effort.

The authors acknowledge the support of the RHIC High Frequency Instrumentation Group in earlier efforts and the C-AD Beam Instrumentation Group in the present effort, and particularly Tom Russo, Tony Curcio, Ron Schroeder, and Al Weston.

And finally, we acknowledge the tireless and efficient oversight of Vadim Ptitsyn in his role as run co-ordinator of RHIC Run 6, as well as the steadfast long-term support and encouragement of Thomas Roser from the very beginnings of this effort.

This work is supported by the U.S. DOE under contract DE-AC02-98CH10886 and the US LHC Accelerator Research Program (LARP).

[1] The tune feedback effort at RHIC was initially a BNL effort, was expanded into a bi-lateral collaboration with CERN in 2002, and became a multi-lab LARP collaboration in 2003. For further information, see http://www.agsrhichome.bnl.gov/LARP/

[2] P. Cameron et al., "RHIC Third Generation PLL Tune Measurement System”, PAC 2003, Portland. http://accelconf.web.cern.ch/AccelConf/p03/PAPERS/ROAB009.PDF

[3] P. Cameron et al., "Advances Toward the Measurement and Control of LHC Tune and Chromaticity", DIPAC 2005, Lyon. http://dipac2005.web.cern.ch/dipac2005/default.htm

[4] Y. Luo et al., "Possible Phase Loop for the Global Decoupling”, PAC 2005, Knoxville. http://accelconf.web.cern.ch/AccelConf/p05/PAPERS/TPAP052.PDF

a much expanded version is available at http://www.agsrhichome.bnl.gov/AP/ap notes/ap note 174.pdf

[5]Y. Luo et al, "Continously measure global difference coupling using a phase-locked-loop tune meter in the RHIC", this journal issue.

[6] M. Gasior and R. Jones, "The Principle and First Results of Betatron Tune Measurement by Direct Diode Detection", LHC-Project-Report-853. http://doc.cern.ch/archive/electronic/cern/preprints/lhc/lhc-project-report-853.pdf

[7] M. Gasior and R. Jones, "High Sensitivity Tune Measurement by Direct Diode Detection”, DIPAC 2005, Lyon. http://dipac2005.web.cern.ch/dipac2005/default.htm

[8] R. Jones et al., “Towards a Robust Phase Locked Loop Tune Feedback System”, ibid.

[9] G. Lambertson and D. Goldberg, “A Primer on Pickups and Kickers”, AIP 249, 1990.

[10] G. Decker, “The Design of Beam Pickups and Kickers”, BIW 1994, Vancouver.

[11] C. Schultheiss et al., "Real Time Betatron Tune Control in RHIC", EPAC 2002, Paris. 
http://accelconf.web.cern.ch/AccelConf/e02/PAPERS/THPRI041.pdf

[12] P. Cameron et al., "The Effects and Possible Origins of Mains Ripple in the Vicinity of the Betatron Spectrum”, DIPAC 2005. Lyon. http://dipac2005.web.cern.ch/dipac2005/default.htm

[13] P. Cameron et al., "Observations of Direct Excitation of the Betatron Spectum by Mains Harmonics in RHIC", C-AD AP note 253, September 2006. This note is available at http://www.agsrhichome.bnl.gov/AP/ap notes/

[14] S. Tepikian et al., "Measuring Chromaticity along the Ramp using the PLL Tune-meter in RHIC", EPAC 2002, Paris .

http://accelconf.web.cern.ch/AccelConf/e02/PAPERS/THPRI075.pdf

[15] S. Fartoukh and J.P. Koutchouk., "On the Measurement of the Tunes, Coupling, and Detunings with Momentum and Amplitude in LHC", Functional Specification LHC-B-ES-004, 2002.

http://www.rhichome.bnl.gov/LARP/papers/LHC tune_spec.pdf 